\title{
First report of probable neurobrucellosis in Australia
}

\begin{abstract}
We report the first known Australian case of probable neurobrucellosis, in a young feral-pig shooter who presented with episodic left-sided visual loss and left-sided numbness and headache. Treatment with intravenous ceftriaxone and oral rifampicin, doxycycline and trimethoprim-sulfamethoxazole resulted in a good clinical response.
\end{abstract}

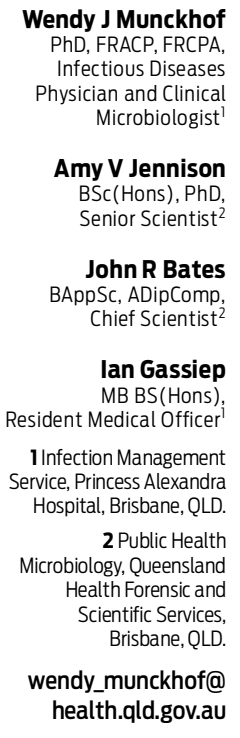

MJA 2013; 199: 423-425 doi: 10.5694/mjal2.11561

\section{Clinical record}

In September 2011, a 29-year-old man from rural western Queensland suddenly developed left-sided visual loss. This lasted 15 minutes and was followed by left leg numbness that spread to his left arm and the left side of his face. This was accompanied by slurred speech and lasted 1.5 hours. He had no loss of power. Afterwards, he regained full function but suffered a 1-hour headache. He was then asymptomatic for 4 days before having another neurological episode, which began with 15 minutes of left leg numbness followed by 15 minutes of left arm numbness. The patient was admitted to our hospital 2 weeks later, after he reported the neurological events to a doctor.

A detailed history revealed the patient worked as a cattle farmer and regularly hunted feral pigs and kangaroos, gutting and skinning the animals himself and frequently lacerating his hands while doing so. In July 2008, he had experienced nausea, vomiting, lethargy and night sweats. At the time, serological tests for Brucella were done: enzyme immunoassay (EIA) results were positive for IgM and negative for IgG, and agglutination showed a total antibody titre of 80 . This was reported as suggestive of early acute brucellosis. The patient was treated with oral doxycycline $100 \mathrm{mg}$ twice daily. He took about 2 weeks of a planned 6-week course, but stopped taking it after his symptoms resolved.

In June 2011, he had presented to his local hospital with a 2-week history of dizziness, nausea, lethargy and night sweats. A diagnosis of brucellosis was made again and he commenced oral doxycycline $100 \mathrm{mg}$ twice daily. After 4 days, he stopped taking it because his condition had not improved. He continued to experience nausea, dizziness and lethargy throughout July and August 2011 but did not seek medical advice.

Apart from the above episodes, the patient had previously been well, and he reported no other history of headache or symptoms suggestive of migraine. He had never travelled outside Australia. At admission, it was noted that the results of serological tests for Brucella that had been done in June 2011 included positive reactions for IgG and IgM on EIA, a total agglutinating antibody titre of $>1280$, and an anticomplementary reaction by complement fixation test (CFT) (which can have several causes, including an extremely high antibody titre or a contaminated specimen).

On admission, the patient looked well and was alert, afebrile and oriented, and no clinical abnormalities were detected on examination. Full blood count, erythrocyte sedimentation rate and chest radiography results were normal. Results of three sets of blood cultures were negative after prolonged incubation. Results of serological tests for HIV, Q fever, dengue, syphilis, Borrelia burgdorferi (Lyme disease agent), Toxoplasma and Leptospira were negative. The patient also tested negative for autoantibodies. Serological tests for Brucella were repeated: EIA results were again positive for IgG and IgM, agglutination showed a total antibody titre of $>2560$, and CFT showed an anticomplementary reaction.

Contrast cerebral magnetic resonance imaging (MRI) and magnetic resonance angiography (MRA), electroencephalography and transthoracic echocardiography results were all normal. Results of lumbosacral contrast MRI, performed because the patient had intermittent chronic lumbar pain, were normal. Cerebrospinal fluid (CSF) pressure was normal and other CSF findings were: protein level, $570 \mathrm{mg} / \mathrm{L}$ (reference interval [RI], 150$500 \mathrm{mg} / \mathrm{L}$ ); glucose level, $2.9 \mathrm{mmol} / \mathrm{L}$ (RI, $2.8-4.0 \mathrm{mmol} / \mathrm{L}$ ); white cell count, $33 \times 10^{6} / \mathrm{L}$ (100\% lymphocytes) (RI, $<5 \times 10^{6} / \mathrm{L}$ ); red cell count, $120 \times 10^{6} / \mathrm{L}$ (RI, $\left.<5 \times 10^{6} / \mathrm{L}\right)$. Results of serological tests for syphilis, testing for cryptococcal antigen, a Gram stain, an acid-fast bacilli stain and CSF cultures (including for mycobacteria, and for fungi after prolonged incubation) were all negative. Three additional tests for Brucella in CSF were performed; these tests have not been well validated on CSF, because neurobrucellosis is rare, but have been validated on other specimens. Results of these were all negative: by agglutination, total antibody titre in CSF was < 10; by CFT, total antibody titre in CSF was <8; and results of both these tests were negative when repeated on diluted CSF (to exclude a prozone effect). Further, Brucella DNA was not detected in CSF in polymerase chain reaction (PCR) assays (Box).

No evidence of ocular brucellosis was found. Given the patient's history and the results of investigations, he was diagnosed with probable neurobrucellosis. He commenced intravenous ceftriaxone $4 \mathrm{~g}$ once daily, oral doxycycline $100 \mathrm{mg}$ twice daily and oral rifampicin $300 \mathrm{mg}$ twice daily. 


\begin{tabular}{|c|c|c|c|c|}
\hline Target & Primer & Reference & Sequence (5'-3') & Limit of detection \\
\hline \multirow[t]{2}{*}{ B. suis } & Universal Forward primer $^{\dagger}$ & 16 & CAT GCG CTA TGA TCT GGT TAC & $200 \times 10^{-12} \mathrm{~g}$ \\
\hline & Reverse primer & 16 & ACC GGA ACA TGC AAA TGA C & \\
\hline \multirow[t]{2}{*}{ B. melitensis } & Universal Forward primer ${ }^{\dagger}$ & 16 & CAT GCG CTA TGA TCT GGT TAC & $20 \times 10^{-15} \mathrm{~g}$ \\
\hline & Reverse primer & 16 & AGT GTT TCG GCT CAG AAT AAT C & \\
\hline \multirow[t]{2}{*}{ B. abortus } & Universal Forward primer ${ }^{\dagger}$ & 16 & CAT GCG CTA TGA TCT GGT TAC & $200 \times 10^{-12} \mathrm{~g}$ \\
\hline & Reverse primer & 16 & GGC TTT TCT ATC ACG GTA TTC & \\
\hline \multirow[t]{2}{*}{ Brucella genus } & BsppF & 17 & GTG GCG ATC TTG TCC G & $20 \times 10^{-12} \mathrm{~g}$ \\
\hline & BsppR & 17 & ACG GCG ATG GAT TTC CG & \\
\hline
\end{tabular}

*A modified high-resolution melt (HRM) real-time reverse transcriptase PCR method was performed on the 7500 Fast Dx Real-Time PCR System (Life Technologies) Using DNA extracted from the patient's CSF, the pairs of primers listed above and MeltDoctor HRM Master Mix (Life Technologies). The PCR steps were: (i) one cycle of $95^{\circ} \mathrm{C}$ for 10 minutes; (ii) 40 cycles of $95^{\circ} \mathrm{C}$ for 15 seconds then $60^{\circ} \mathrm{C}$ for 1 minute; (iii) one dissociation step. ${ }^{1,2} \mathrm{However}$, the Brucella targets were not detected. † The Universal Forward primer sequence used was a modified version of that published by Redkar et al. ${ }^{1}$

Oral amitryptyline (25 $\mathrm{mg}$ at night) was given because the major neurological manifestation was the episodic leftsided hemianaesthesia, which appeared to have a migrainous component. The patient was observed in hospital for 9 days and was asymptomatic - without fever, headache or further neurological events. He was discharged to a relative's house in Brisbane on a regimen of home intravenous ceftriaxone and oral doxycycline and rifampicin for 4 weeks.

At outpatient review 4 weeks after discharge, the patient had remained asymptomatic. He was switched from intravenous ceftriaxone to oral co-trimoxazole (trimethoprim $160 \mathrm{mg}$ plus sulfamethoxazole $800 \mathrm{mg}$ ) twice daily. Doxycycline and rifampicin were continued. The patient returned to his home in rural western Queensland. At outpatient reviews 3, 6, 9 and 12 months after discharge, he was well and asymptomatic. Doxycycline, rifampicin and co-trimoxazole were discontinued 12 months after discharge. Three months later, he remained well. Results of serological tests for Brucella were still positive for IgG and IgM by EIA, but his total serum antibody titre by agglutination (which had peaked at $>2560$ at the time of the acute neurological symptoms) was 40 . The anticomplementary reaction by CFT remained.

\section{Discussion}

To our knowledge, this is the first human case of probable neurobrucellosis acquired in Australia. The differential diagnoses considered included new-onset migraine, transient ischaemic attacks, vasculitis, neurosyphilis, cryptococcal or tuberculous meningitis, and Lyme disease. Our patient was young and had no past history of migraine or risk factors for cerebrovascular disease. He tested negative for autoantibodies and had lymphocytic CSF, but he tested negative for cryptococcal disease, syphilis, tuberculosis and Lyme disease, and he had never travelled outside Australia.

Brucellosis is rare in Australia, with 21 cases reported nationwide in $2010 .{ }^{3}$ About $80 \%$ of cases occur in Queensland, mostly in young men who shoot and gut feral pigs. ${ }^{4}$ Swine brucellosis (caused by Brucella suis) is endemic in feral pigs in Queensland, but bovine brucellosis (caused by Brucella abortus) was eradicated from Australian cattle in 1989. ${ }^{4}$
Direct invasion of the central nervous system (CNS) occurs in fewer than $5 \%$ of brucellosis cases. Our patient met all four of the diagnostic criteria for brucellar meningitis or meningoencephalitis. ${ }^{5}$ Most CNS brucellosis cases occur in Middle Eastern countries, including Turkey, Saudi Arabia and Iran. ${ }^{5-7}$ Brucella melitensis and B. abortus, the most common Brucella species in the Middle East, do not naturally occur in Australia. Occasional Australian cases of acute $B$. melitensis infection occur due to consumption of imported unpasteurised goat and sheep cheeses. Based on the known epidemiology of brucellosis in Australia, we suspect that our patient had B. suis infection, as he had a history of exposure to feral pigs and no history of consumption of imported cheeses.

The most common clinical manifestation of neurobrucellosis is an acute or chronic lymphocytic meningitis. ${ }^{5-7}$ Cerebral mycotic aneurysms have been reported, often as a consequence of embolic phenomena from brucellar endocarditis, and can rupture with catastrophic results. ${ }^{8,9}$ For this reason, and because both episodes of neurological deficit occurred in the same cerebrovascular territory, MRA and echocardiography were performed. Neurobrucellosis can also cause a cerebral arterial or venous inflammation with resultant lacunar infarcts, small haemorrhages or venous thromboses. ${ }^{9}$ If the inflammatory lesion is small, it can be missed on MRI and MRA, particularly in early lacunar infarction. We considered repeating MRI and MRA at a later date, but the patient was not keen to do this in the absence of further symptoms. We think it most likely that our patient had this manifestation of neurobrucellosis, with negative results from early radiological testing as the lesion was too small to detect. As the results of CSF culture are positive in fewer than $20 \%$ of neurobrucellosis cases, ${ }^{6}$ diagnosis was, until recently, made by detecting antibodies in the CSF of patients with serologically confirmed brucellosis and clinical syndromes suggestive of neurobrucellosis. ${ }^{6}$ However, in patients who have very high serum antibody titres and for whom there is a high clinical suspicion of neurobrucellosis, CSF antibody titres are often low, or antibodies are not detected in CSF. ${ }^{10}$ This low sensitivity, and the advent of molecular detection techniques, has led to the development of PCR assays for detecting DNA from Brucella species. In one report, PCR results were positive for all six 
patients with suspected neurobrucellosis (CSF culture and CSF serological tests produced positive results for only two and four patients, respectively). ${ }^{11}$ Our patient had very high serum antibody levels and a lymphocytic meningitis but we could not detect Brucella in his CSF by culture, agglutination, CFT or PCR. However, there is no specific literature on diagnosis of neurobrucellosis caused by $B$. suis, the species native to Australia (although this species also occurs in other countries).

Neurobrucellosis treatment must include antibiotics that cross the blood-brain barrier well. ${ }^{6,7,12-14}$ Combination therapy with at least two of doxycycline, rifampicin and cotrimoxazole is recommended. Aminoglycosides do not penetrate the CSF well, so, unlike for other forms of brucellosis, these are not usually used. ${ }^{12}$ Ceftriaxone has been shown to have good activity against most strains of $B$. melitensis, ${ }^{14,15}$ although there are no reports of its activity against $B$. suis. Also, we could not test antibiotic susceptibility because we were unable to culture Brucella. Ceftriaxone achieves CSF levels 10-40 times higher than the minimum inhibitory concentration for B. melitensis, so is an attractive choice for therapy. However, therapeutic failure is common unless ceftriaxone is used as part of combination therapy. ${ }^{12,16}$

Duration of therapy for neurobrucellosis is usually at least 6 months. ${ }^{6,7,12,13}$ We empirically chose 12 months as the treatment duration based on available literature. A subsequently published retrospective review of three different combination regimens used to treat 215 patients with neurobrucellosis in Turkey suggests that 6 months would have been sufficient, particularly if ceftriaxone was used, as patients treated with this agent had a better outcome. ${ }^{13}$ The other antibiotics considered in this review were oral doxycycline, rifampicin and co-trimoxazole. Finally, corticosteroids are sometimes used to treat neurobrucellosis, but these appear to be most useful in patients with raised intracranial pressure, papilloedema or polyneuropathies. ${ }^{12}$ Our patient had none of these features.

Brucellosis still occurs in Australia. Although it has been eradicated from Australian cattle, clinicians should consider the diagnosis in shooters of feral pigs and kangaroos, particularly in northern Australia. This case illustrates the risks of progression to chronic brucellosis if patients do not complete the recommended 6-week antibiotic course for acute disease. ${ }^{17}$ To our knowledge, there are no current public health programs in Australia to educate feral pig shooters about the risks of acquiring infection.

\section{Competing interests: No relevant disclosures.}

Received 19 Oct 2012, accepted 31 Jul 2013.

1 Redkar R, Rose S, Bricker B, DelVecchio V. Real-time detection of Brucella abortus, Brucella melitensis and Brucella suis. Mol Cell Probes 2001; 15: 43-52.

2 Winchell JM, Wolff BJ, Tiller R, et al. Rapid identification and discrimination of Brucella isolates by use of real-time PCR and high-resolution melt analysis. J Clin Microbiol 2010; 48: 697-702.

3 NNDSS Annual Report Writing Group; Milton A, Stirzker S, Trungove M, et al. Australia's notifiable disease status, 2010: annual report of the National Notifiable Diseases Surveillance System. Commun Dis Intell Q Rep 2012; 36: 1-69.

4 Queensland Government. Brucellosis: Queensland Health guidelines for public health units. Version 1.0, August 2010. http://www.health.qld.gov.au/cdcg/ index/brucell.asp (accessed Apr 2013).

5 Erdem H, Kilic S, Sener B, et al. Diagnosis of chronic brucellar meningitis and meningoencephalitis: the results of the Istanbul-2 study. Clin Microbiol Infect 2013; 19: E80-E86.

6 Gul HC, Erdem H, Bek S. Overview of neurobrucellosis: a pooled analysis of 187 cases. Int J Infect Dis 2009; 13: e339-e343.

7 Haji-Abdolbagi M, Rasooli-Nejad M, Jafari S, et al. Clinical and laboratory findings in neurobrucellosis: review of 31 cases. Arch Iran Med 2008; 11: 21-25.

8 Hansmann GH, Schenken JR. Melitensis meningo-encephalitis: mycotic aneurysm due to Brucella melitensis var porcine. Am J Pathol 1932; 8: 435-444.

9 Inan AS, Ceran N, Erdem I, et al. Neurobrucellosis with transient ischemic attack, vasculopathic changes, intracerebral granulomas and basal ganglia infarction: a case report. J Med Case Rep 2010; 4: 340.

10 Sanchez-Sousa A, Torres C, Campello MG, et al. Serological diagnosis of neurobrucellosis. J Clin Pathol 1990; 43: 79-81.

11 Colmenero JD, Queipo-Ortuño MI, Reguera JM, et al. Real time polymerase chain reaction: a new powerful tool for the diagnosis of neurobrucellosis. J Neurol Neurosurg Psychiatry 2005; 76: 1025-1027.

12 Pappas G, Akritidis N, Christou L. Treatment of neurobrucellosis: what is known and what remains to be answered. Expert Rev Anti Infect Ther 2007; 5: 983-990.

13 Erdem H, Ulu-Kilic A, Kilic S, et al. Efficacy and tolerability of antibiotic combinations in neurobrucellosis: results of the Istanbul study. Antimicrob Agents Chemother 2012; 56: 1523-1528.

14 Bosch J, Liñares J, López de Goicoechea MJ, et al. In-vitro activity of ciprofloxacin, ceftriaxone and five other antimicrobial agents against 95 strains of Brucella melitensis. J Antimicrob Chemother 1986; 17: 459-461.

15 Palenque E, Otero JR, Noriega AR. In vitro susceptibility of Brucella melitensis to new cephalosporins crossing the blood-brain barrier. Antimicrob Agents Chemother 1986; 29: 182-183.

16 Lang R, Dagan R, Potasman I, et al. Failure of ceftriaxone in the treatment of acute brucellosis. Clin Infect Dis 1992; 14:506-509.

17 Antibiotic Expert Group. Therapeutic guidelines: antibiotic. Version 14. Melbourne: Therapeutic Guidelines Limited, 2010. 